\title{
Meta-Brokering Solutions for Expanding Grid Middleware Limitations
}

\author{
Attila Kertész ${ }^{1}$, Ivan Rodero ${ }^{2}$, and Francesc Guim² \\ 1 MTA SZTAKI Computer and Automation Research Institute \\ H-1518 Budapest, P. O. Box 63, Hungary \\ attila.kertesz@sztaki.hu \\ 2 Technical University of Catalonia (UPC) \\ Jordi Girona 29, 08034 Barcelona, Spain \\ \{irodero, fguim\}@ac.upc.edu
}

\begin{abstract}
Grid Resource Management tools evolved from manual discovery and task submission to sophisticated brokering solutions. User requirements created certain properties that resource managers have learned to support. This development is still continuing, and users already need to stress themselves to distinguish brokers and migrate their applications when they move to a different grid. Moreover, grid interoperability have emerged the need for higher level brokering services. This paper introduces a meta-brokering approach that means a higher level resource management by enabling automatic and simultaneous utilization of Grid Brokers. First we gather the requirements of this novel middleware service then define a general meta-brokering architecture. Finally we show how meta-brokers can be implemented in different grid environments and we conclude with their evaluations.
\end{abstract}

Keywords: Grid Interoperability, Grid Meta-brokering, Grid Scheduling, Grid Services.

\section{Introduction}

In the last decade grid resource management has evolved from manual discovery and task submission to sophisticated brokering solutions. Nowadays research directions are focusing more on user needs: more efficient utilization and interoperability play the key roles. Grid resource management is probably the research field most affected by user demands. Though well-designed, evaluated and widely used resource brokers, meta-schedulers have been developed, new capabilities are still required, such as agreement and interoperability support. These two directions also depend on other grid middleware capabilities and services, and since they cannot cross the border of these middleware solutions, they need significant changes affecting the whole system. Trying to enlarge the limitation borders, in this paper we introduce a meta-brokering approach as an evolutionary step for grid resource management, which does not need radical changes to the whole system. The need for interoperability among different grid systems has raised 
several questions and directions. The advance of grids seems to follow the way envisaged and assigned by the Next Generation Grids Expert Group [1]. The SOKU architecture [1 enables more flexibility, adaptability and advanced interfaces, therefore interoperability is evident and congenital in these systems. Moving towards this direction two works envisaged WWG (World Wide Grid) as the next generation of grids in a similar manner as the Internet was born. One of the first vision is the InterGrid [2, which promotes interlinking of grid systems through peering agreements to enable inter-grid resource sharing. This approach is a more economical view, where business application support is a primal goal, and this also supposed to establish sustainability. The second approach is detailed in 3 which states that three major steps are required to create the WWG architecture: existing production grids should be connected by uniform meta-brokers, workflow-oriented portals should interface these meta-brokers, and a repository of workflow grid services should be created. As a final solution the inter-connected meta-brokers should solve the interoperability among different grids through uniform interfaces. Both visions proceed from the current grid architectures and conclude in a more or less redesigned one.

In this paper we gather the requirements of this novel middleware service that define a general meta-brokering architecture. Moreover, we show how metabrokers can be implemented in different grid environments. Finally, we evaluate our implementations in two diferent simulation environment, and demonstrate that the meta-brokering approach can reduce execution time and improve the overall interoperable system performance. The main contributions of this work are the design, implementation, and evaluation of the meta-brokering approach in two different scenarios.

The rest of the paper is organized as follows. In Section 2 we present the related work in higher level brokering directions. In Section 3, we introduce a general meta-brokering architecture by gathering its requirements. In Section 4, we show how the presented architecture can be realized in two different grid user environments, and we describe the simulation environments used for evaluating these realized solutions. Finally, in Section 5, we present the conclusions and future work.

\section{Related Work}

Several research groups have noticed interoperability problems at the level of grid resource management. One of the promising approaches aimed at enabling communication among existing resource brokers. The GSA-RG of OGF [15] is currently working on a project enabling grid scheduler interaction. They plan to define common protocol and interface among schedulers enabling inter-grid usage. In this work they propose a Scheduling Description Language to extend the currently available job descriptions. Another instance of this approach is the Latin American Grid (LA Grid) initiative. The meta-scheduling project in LA Grid [16] aims to support grid applications with resources located and managed in different domains. They define meta-broker instances with a set of functional 
modules: connection management, resource management, job management and notification management. These modules implement the APIs and protocols used in LA Grid through web services. Each meta-broker instance collects resource information from the other instances having a view of all resources in aggregated form. The Koala grid scheduler [4] was designed to work on DAS-2 interacting with Globus middleware services with the main features of data and processor co-allocation, lately it is being extended to support DAS-3 and Grid'5000. To inter-connect different grids, they have also decided to use inter-broker communication. Their policy is to use a remote grid only if the local one is saturated. In an ongoing experiment they use a so-called delegated matchmaking (DMM), where Koala instances delegate resource information in a peer-2-peer manner. Gridway introduces a Scheduling Architectures Taxonomy [5, where they describe a Multiple Grid Infrastructure. It consists of different categories, we are interested in the Multiple Meta-Scheduler Layers, where Gridway instances can communicate and interact through grid gateways. These instances can access resources belonging to different administrative domains (grids/VOs). The basic idea is to pass user requests to another domain when the current is overloaded. This approach follows the same idea as the previously introduced DMM.

Comparing the previous approaches, we can see that all of them use a new method to expand current grid resource management boundaries. Meta-brokering appears in a sense that different domains are being examined as a whole, but they rather delegate resource information among domains, broker instances or gateways. Usually the local domain is preferenced, and when a job is passed to somewhere else, the result should be passed back to the initial point. In the next section we focus on a solution that utilizes and delegates broker information by reaching the brokers through their current interfaces.

\section{General Meta-Brokering Architecture}

We have developed solutions to make resource managers' data available for cooperated, automatic processing in the form of metadata. We provide language schemas to store and share this metadata, and to be processed by various scheduling policies. Using these advanced techniques we present a meta-brokering architecture that enables a higher level brokering that has a global, up-to-date view of broker capabilities and availability. Figure 1] is intended to show all the components and tools needed by a General Meta-Broker. In the following we describe these components by introducing the main requirements of this higher level brokering service:

- JDL - Job Description Language: Since the goal of the meta-brokering service is to offer a uniform way to access various grids, a unified description format is needed to specify all the user requirements.

- CDL - Capability Description Language: Each broker has a different set of functionalities, they can be specialized in different application-types. In order to store and track these properties, it is required to use a CDL. 


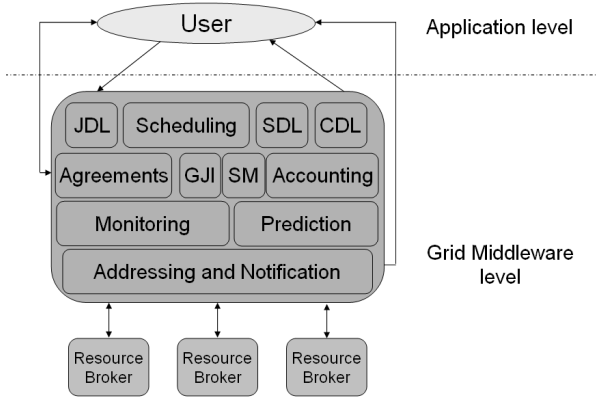

Fig. 1. Required components and tools of a General Meta-Broker

It should be general enough to include all the service capabilities (interfaces, job submission, monitoring and agreements).

- SDL - Scheduling Description Language: Besides CDL and JDL the scheduling requirements and policies also need to be stored separately. The users can express their needs by extending the JDL with SDL, and the scheduling policies and methods of the brokers can be stored in this format.

- Scheduling: This component performs the scheduling (matchmaking) of incoming user requests. A proper grid broker (which implies a domain, VO or execution environment) needs to be selected for a user job taking into account the available scheduling policies.

- GJI - Global Job Identifiers: It is important to have unique mapping of user jobs to different grids. An implementation can be a single job ID provider for the meta-brokering system or simply using each broker system as a prefix for the assigned grid job ID.

- SM - Security Management: The role of this component is to provide secure access to the interconnected domains. For example, different user certificates, proxies may be accepted in different VOs and grids. Providing a transparent way for users these various proxies also need to be handled by the Meta-Broker. (In most cases we rely on the underlying Grid Security Infrastructure (using proxies).)

- Accounting Mechanism: The GJI and SM can be a part of a global accounting component. The role of this mechanism is to manage user access by pre-defined policies. Though grid economy is still in a pre-mature state, in the future the meta-brokering service should also serve business grids.

- Agreements Mechanism: This component is in connection with the Accounting mechanism. Service Level Agreements (SLA) are planned to be used in future generation grids. The role of this part is to negotiate user requirements, which can also affect scheduling policies. When agreements will be generally accepted and used, this mechanism should be extended to do negotiations with the brokers.

- Monitoring Mechanism: Reliable operation requires global monitoring, in terms of the inter-connected brokers, reachable domain, grid resources and 
loads, and local component functionalities. Self-awareness and fault tolerance need to be provided by the system itself, which needs extensive monitoring.

- Prediction Mechanism: This component is in connection with the Monitoring and Scheduling mechanisms. It is necessary to perform calculations of broker availability, service utilization and user request load to cope with the highly dynamic nature of grids.

- Addressing and Notification Mechanism: This component is responsible for accessing the inter-connected resource brokers, and managing communication including local events and external job state notifications.

In addition to the architecture model and the required interfaces it is important to deal with scheduling at the meta-brokering level. Based on the components of the architecture shown in Figure 1, we propose a general meta-brokering policy relying on the capabilities and measured performances of the utilized brokers. The selection of the appropriate brokering system can be done using a multi-criteria algorithm that can take into account the gathered and predicted metadata stored in SDL and CDL. The general scheduling policy examines the job description (JDL) and matches its requirements to the metadata stored in CDL and SDL. The matchmaking algorithm consists of the following steps: In the first phase the basic job requirement attributes and the scheduling constraints are matched against the basic broker capabilities: this selection determines a group of brokers that are able to fulfill the job request. In this phase those brokers are kept that are able to submit the specified job type and work with the requested middleware services. In the second phase the brokers are filtered by all the scheduling requirements of the user job. For all of the brokers that could pass these filtering phases a rank is computed by the actual broker performance and domain load. For the brokers that could only pass the first phase reduced ranks are assigned. Finally the list of the brokers is ordered by these ranks. The broker with the highest rank is selected for submission. Different scheduling policies can be defined regarding the data stored in SDL. It also depends on the deployed architecture, which policies are implemented. In economy-based grid environments scheduling policies could rely on the agreements and accounting information to match user budget and maximize domain earnings. An ongoing work in UPC investigate to use policies based on historical job/resource information to make scheduling decisions. Later we can use the achievements of this approach to improve the meta-brokering algorithm and create another policies with estimation and prediction results.

\section{Meta-Broker Architecture Realizations}

Having all the requirements defined, we are able to implement and build a metabrokering service. As we stated in the previous section, semantics are crucial to establish interoperability. Standardization should be taken into account during the design and development of sustainable solutions. To walk on this way, we use the standards and widespread technologies, where applicable. To describe 
user jobs we use JSDL (Job Submission Description Language) [17] and WSAgreement for handling agreements. Regarding CDL we developed a language called BPDL (Broker Property Description Language) 8], which had also incorporated SDL. To provide full support for our meta-brokering approach we have revised and modified BPDL and gathered the scheduling-related attributes to MBSDL (Meta-Broker Scheduling Description Language). The structure of the new BPDL - that we call BPDL 2.0 -, remains nearly the same, we have only clarified some attributes, added missing ones and separated the schedulingrelated ones to MBSDL. The updated BPDL version includes: BrokerID, Interface (to providee metadata about the accessibility and notification), Monitoring, Security, and Middleware (kind of middleware, grid or VO the broker can operate), JobType, RemoteFileAccess. We use the PerformanceMetrics field to store how successfully the broker performed job requests, and how reliable its services are. The Prediction attribute can be used to store predicted data about broker availability and reliability. The MBSDL language can be used to extend BPDL with scheduling related attributes. Since JSDL is also lacking these attributes, MBSDL can also be regarded as a JSDL extension. Its schema contains three fields: Constraints (that have to be fulfilled during scheduling), QoS (Quality of Service) requirements, and Policy (for scheduling policies). Taking into account these ideas, in the following, we present two different realizations of the presented meta-brokering approach. The first one is the Grid Meta-Broker that has been evaluated in a GridSim-based environment, and the second one is the extension of eNanos that has been evaluated with the Alvio simulation framework.

\subsection{The First Solution: Grid Meta-Broker}

The first realization, the Grid Meta-Broker [7], will be used to solve metabrokering in the future version of P-GRADE Portal [6]. Nevertheless it has been designed as a standalone, standards-based Web Service, therefore it is grid middleware and portal independent. It has been derived from the architecture shown in Figure 1]. This service consists of the following components (Figure 2): the Translator is responsible for translating the JSDL of the user job to the language of the appropriate broker that the Grid Meta-Broker selects for submission. The users can use the MBSDL to specify scheduling related attributes, and the Meta-Broker uses BPDL together with MBSDL to store broker properties. The Information Collector (IC) component stores the data of the reachable brokers and historical data of the previous submissions in BPDL. The load of the resources behind the brokers is also taken into account to help the MatchMaker component to select the proper environment for the submitted job. There are IS Agents reporting to the IC, which regularly check the load of the underlying grids of each connected resource broker. The previously introduced language attributes are used for matching the user requests to the description of the interconnected brokers: which is the role of the MatchMaker component. The Invokers are broker-specific components: they communicate with the interconnected brokers, invoking them with job requests and collecting the results. In the JSDL extension, the middleware constraint field can be used to specify 


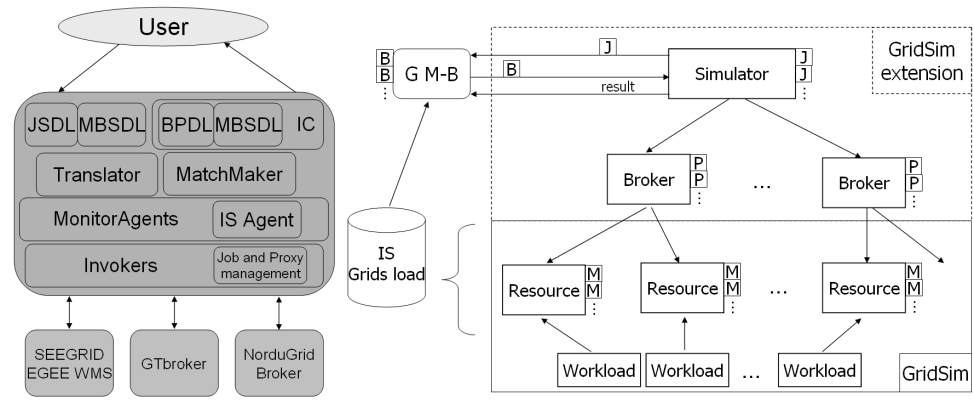

Fig. 2. Grid Meta-Broker architecture (on the left), and its simulation architecture with GridSim (on the right)

proxy names for grids/VOs. This information is used by the Invokers to select the valid proxy from the uploaded files for the actual job submission.

We have created a prototype that is mature enough to perform evaluation test in a simulated environment. We have chosen the GridSim toolkit 9 for our simulations, because it is one of the most widely used and accepted grid simulation tools. It can be used for simulating and evaluating VO-based resource allocation, workflow scheduling, and dynamic resource provisioning techniques in global Grids. It supports modeling and simulation of heterogeneous Grid resources, users, applications, brokers and schedulers in a Grid computing environment. It provides primitives for the creation of jobs (called gridlets), mapping of these jobs to resources, and their management, therefore resource schedulers can be simulated to study scheduling algorithms. GridSim provides a multilayered design architecture based on SimJava [10, a general purpose discrete-event simulation package implemented in Java. It is used for handling the interaction or events among GridSim components. All components in GridSim communicate with each other through message passing operations defined by SimJava.

Our general simulation architecture can be seen in the right part of Figure 2. On the right-bottom part we can see the GridSim components used for the simulated grid systems. Resources can be defined with different grid-types. Resources consist of more machines, to which workloads can be set. On top of this simulated grid infrastructure we can set up brokers. Brokers are extended GridUser entities: they can be connected to one or more resources, they report to the IS Grid load database (which has a similar purpose as a grid Information System), different properties can be set to these brokers (agreement handling, co-allocation, advance reservation, etc.), properties can be marked as unreliable, different scheduling policies can be set for each broker, and resubmission is used, when a job fails due to resource failure. The Simulator (User in the real world) is an extended GridSim entity: it can generate a requested number of gridlets (jobs) with different properties, start and run time (length). It is connected to the brokers and able to submit jobs to them, the default job distribution is the random broker selection (though at least the middleware types are taken into 


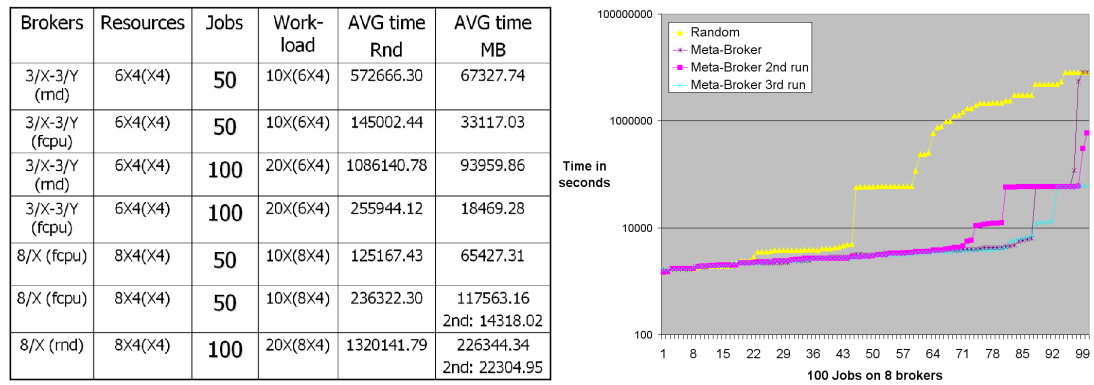

Fig. 3. Grid Meta-Broker evaluation results

account). In case of job failures a different broker is selected for job submission. It is also connected to the Grid Meta-Broker (denoted with G M-B in the figure) through its web service interface and able to call its matchmaking service. Before the Meta-Broker is used for broker selection, it have to be configured with BPDL descriptions, and the job requests need to be submitted in JSDL.

The first evaluation environment consisted of 6 brokers operating on four resources each. Each resource had four machines. Three brokers used resources with GRID_X middleware and the other three used resources with GRID_Y middleware. Four properties were distributed among the brokers, each broker had one unreliable property ( $50 \%$ failure) (this usually happens in real Grids). Half of the jobs were set to GRID_X, the rest to GRID_Y. Each job had only one property and all the four properties were distributed equally among the jobs. The run time of the jobs took around 5 minutes each. 20 percent of the jobs had no special property, the rest were assigned to two properties out of the four (they were distributed equally). We evaluated four different scenarios, Figure 3 shows a table with their parameters. The first line means all the brokers used random selection policy, 50 jobs were submitted into the system and 10 jobs were submitted to each resource as background workload. We used the cleaned SDSC Blue Horizon workload logs from the Parallel Workloads Archive 13. The fifth column shows the average simulation run time of the jobs, when we used random distribution among the brokers. The sixth column shows the average simulation run time for the jobs submitted to brokers selected by the Meta-Broker. Due to the broker property distribution in the first simulation setup, a job with a special property running on either middleware could surely successfully run only on one specific broker. This caused overloading of some brokers even with the use of the Meta-Broker, therefore we created a different environment. The second time we set up 8 brokers operating again on 4-4 resources, but all having the same GRID_X middleware. The same property distribution was used for the jobs. The brokers had 2-2 special properties again, but every second broker had one unreliable property (in this case two brokers could run some job the same time without any failures). In Figure 3 the last two lines contain additional information in their last columns. This means we repeated the measurement again, in a way that the brokers were aware of previous broker failures. In these 
cases the first run can be regarded as a teaching phase, therefore we measured better performance.

On the right of Figure 3, we highlighted a diagram of the last evaluation phase, which best demonstrates the difference of simulation run time with and without the use of the Meta-Broker. The results show that the Grid Meta-Broker provides less execution time by automating broker and grid selection for users. During utilization it is able to adapt to broker failures and to avoid selecting overloaded grids. Future work aims at enhancing its matchmaking algorithm and introducing teaching phases for better adaptation.

\subsection{The Second Solution: Meta-Brokering in HPC Centers with eNANOS}

In our past research we have implemented a brokering approach within the eNANOS Resource Broker [11. It performs the job scheduling based on FCFS and the resource selection based on the matchmaking approach. It also uses hard user criterion (requirements) and soft ones (recommendations) with a predefined set of priorities for computing ranks (the resource with the highest rank is selected for submission). Recently, this scheduling policy has been modelized in the broker layer of Alvio. The Alvio Simulator is a $\mathrm{C}++$ event driven simulator that has been designed and developed to evaluate scheduling policies in high performance architectures. It provides ways to evaluate environments from local centers to meta-brokering solutions. Regarding the local architecture models, like other simulators, given a workload and an architecture definition, it is able to simulate how the jobs would be scheduled using a specific scheduling policy (such as FCFS, or Backfilling policies). The main contribution of this simulator at this level is that it does not only allow modelling the job workflow in the system, but also allows simulating different resource allocation policies.

The other contribution of this simulator is the modelling of resource usage with different jobs running in the system. The researcher is able to specify the setup of the resources available in the architecture (eg. the amount of memory bandwidth in each node) and the amount of resources to be used by the workload jobs. In scenarios for distributed architectures composed by several HPC centers, the simulator allows to simulate multi-site systems (also known as brokering system) and meta-brokering systems. Figure 4 presents the different components that are instantiated inside the simulator in the meta-brokering scenario. The meta-system entity is a conceptual component that models the different elements that are included in the meta-environment. It can contain a centralized meta-broker scheduling entity which potentially can implement centralized-based policies (which is illustrated in Figure 4). It also allows to evaluate $\mathrm{P} 2 \mathrm{P}$ meta-brokering strategies. This meta-system component contains a set of different virtual organization (VO) elements. Each of them contains a global broker entity that is responsible for managing the jobs that users submit to the VO. Furthermore, it contains a set of centers that model the typical HPC local resources introduced previously. Thus, the different centers of the local-scenarios of the simulation model are also instantiated. For each of the 


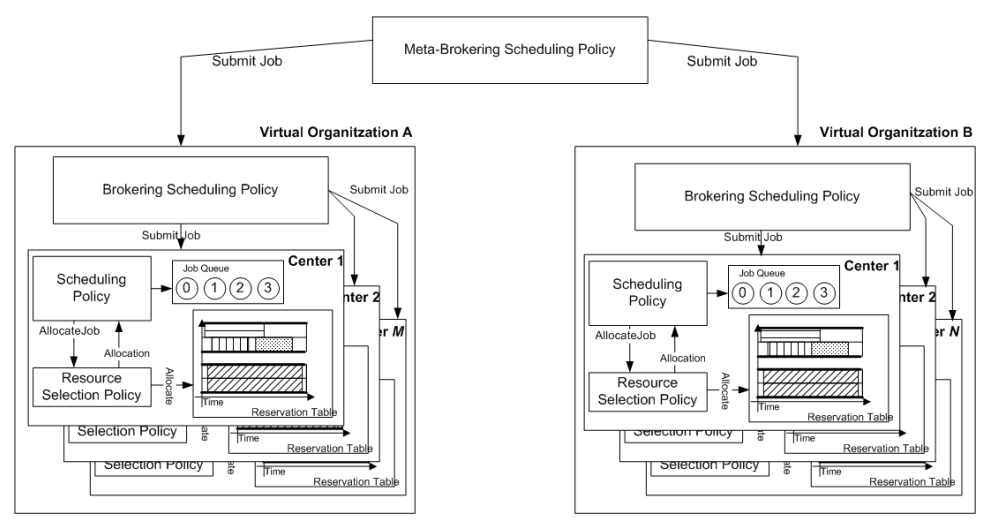

Fig. 4. Meta-brokering Model with a Centralized Approach

center components a local scheduling policy, a resource selection policy and the reservation table are created.

Similarly, we have proposed and implemented a prototype of the BestBrokerRankPolicy. It basically selects the most appropiate broker to submit a job based on a set of rank values corresponding to the different brokers rather than using the local resource information. But for obtaining these values it can consider resource information in an aggregated form and apply an impact factor to each attribute. Since we consider forwarding jobs between brokers, we also consider the average slowdown of the brokers as a QoS metric. An example of the resource aggregated form can be found in 12 and the policy will be widely described and evaluated in a separate paper. In the evaluation we have used traces from the Grid Workloads Archive [14. In particular we have used the workloads from the DAS-2, Grid5000, and Sharcnet systems. We have selected some weeks of each workload trace (around 10.000 jobs in total) with a maximum demand of 512 CPUs and avoiding failed jobs. We found that the average execution time of the jobs depends on the workload, but in average a simulation took around 2 hours. We have defined 3 simulation environments, one for each workload with its own broker instance. For the DAS-2 system we modelized 5 resources with a total of $336 \mathrm{CPUs,} 10$ resources with a total of 574 CPUs for the Grid5000 system, and 10 resources with a total of 3200 CPUs for the Sharcnet system. We have reduced the inter-arrival times for the jobs by a factor of two. This has allowed us to increase the pressure to the system by incrementing the load. In the evaluation we have simulated four different scenarios. First, we simulated the scenario where each virtual organization schedules only their own jobs. Thus we evaluated the performance of each of the different virtual organizations without any connection among them. For the second time, we simulated interconnected VOs using the same workloads. Thereby, we have evaluated the effect of the job forwarding between the different grid brokers in a P2P fashion.

Table 1 shows the performance results for the four different scenarios. We present the total workload execution time, and the $95^{\text {th }}$ percentile and average 
Table 1. Evaluation results with Alvio

\begin{tabular}{|c|c|c|c|c|}
\hline Workload & Exec Time (h) & Avg Wait (s) & Avg SLD & CPU Util \\
\hline \hline DAS-2 & 1,486 & 1,789 & 11,26 & $28(8,33 \%)$ \\
Grid5000 & 1,565 & 4,850 & 13,06 & $39(6,79 \%)$ \\
Sharcnet & 1,596 & 3,011 & 15,78 & $200(6,25 \%)$ \\
\hline Overall & 1,596 & 3,216 & 13,36 & $267(7,12 \%)$ \\
\hline \hline Meta-brokering & 1,410 & 274 & 1,34 & $314(7,63 \%)$ \\
\hline
\end{tabular}

metrics for the wait time, slowdown and CPU utilization. The first three rows of the table show the performance values for the three VOs. In all the three scenarios we can notice that the processor utilization is relatively small. The Grid5000 and Scharnet show similar values in terms of execution time and CPU utilization. The DAS-2 shows substantially lower execution time, wait time, and slowdown. The third row of the table shows the average of all the variables considering all the three VOs. In the last row of the table, that contains the metabrokering approach, the system performance is around $11 \%$, which is better then considering the job forwarding. When the P2P meta-brokering is enabled the average wait time is reduced by a factor of 11 times. Futhermore, the slowdown is also reduced by a factor of 10 times. Thus these results indicate that this approach potentially can provide good performance in large scenarios.

\section{Conclusions and Future Work}

In this paper we have shown the ongoing research directions in the field of grid resource management and stated the necessary steps to be taken to establish a higher level of grid interoperability with meta-brokering by expanding current middleware limitations. We also have defined the essential requirements of a novel grid middleware service called Meta-Broker, we have proposed a general architecture, and we have shown how it can be realized in two different grid environments. Finally, we have developed simulators to these environments to evaluate our implemented prototypes with different workload samples. The results of our simulation-based evaluations show that our meta-brokering approach has a significant gain compared to the approach based on independent brokering systems. In particular, we have measured less total job execution times and identified significant improvement of the overall interoperable systems performance. Our future work aims at investigating new meta-brokering scheduling policies with SLA usage and enlarging the simulation scenarios to evaluate grid environments with more brokers. We also plan to validate our approach in real execution environments such as the P-GRADE portal and the LA Grid infrastructure.

\section{Acknowledgement}

This research work was partly supported by the FP6 Network of Excellence CoreGRID funded by the European Commission (Contract IST-2002-004265) and by the Spanish Ministry of Science and Technology under contract TIN2007-60625. 


\section{References}

1. Next Generation Grids Report: Future for European Grids: GRIDs and Service Oriented Knowledge Utilities - Vision and Research Directions 2010 and Beyond (NGG3) (December 2006)

2. Assuncao, M.D., Buyya, R., Venugopal, S.: InterGrid: A Case for Internetworking Islands of Grids. In: Concurrency and Computation: Practice and Experience (CCPE), July 2007. Wiley Press, New York (2007)

3. Kacsuk, P., Kiss, T.: Towards a scientific workflow-oriented computational World Wide Grid. CoreGRID Technical Report TR-115 (December 2007)

4. Iosup, A., Epema, D.H.J., Tannenbaum, T., Farrellee, M., Livny, M.: InterOperating Grids through Delegated MatchMaking. In: Supercomputing 2007 (SC 2007), Reno, Nevada (November 2007)

5. Vazquez, T., Huedo, E., Montero, R.S., Llorente, I.M.: Evaluation of a Utility Computing Model Based on the Federation of Grid Infrastructures. In: Kermarrec, A.-M., Bougé, L., Priol, T. (eds.) Euro-Par 2007. LNCS, vol. 4641, pp. 372-381. Springer, Heidelberg (2007)

6. Kacsuk, P., Sipos, G.: Multi-Grid, Multi-User Workflows in the P-GRADE Grid Portal. Journal of Grid Computing, 1-18 (Feburary 2006)

7. Kertesz, A., Kacsuk, P.: Meta-Broker for Future Generation Grids: A new approach for a high-level interoperable resource management. In: CoreGRID Workshop on Grid Middleware in conjunction with ISC 2007 conference, Dresden, Germany (June 2007)

8. Kertesz, A., Rodero, I., Guim, F.: Data Model for Describing Grid Resource Broker Capabilities. In: CoreGRID Workshop on Grid Middleware in conjunction with ISC 2007 conference, Dresden, Germany, June 25-26 (2007)

9. Buyya, R., Murshed, M.: GridSim: A Toolkit for the Modeling and Simulation of Distributed Resource Management and Scheduling for Grid Computing. Concurrency \& Computation: Prac. \& Exp. (November-December 2002)

10. Howell, F., McNab, R.: SimJava: A discrete event simulation library for Java. In: Intl. Conference on Web-Based Modeling and Simulation, San Diego, USA (1998)

11. Rodero, I., Corbalán, J., Badía, R.M., Labarta, J.: eNANOS grid resource broker. In: Sloot, P.M.A., Hoekstra, A.G., Priol, T., Reinefeld, A., Bubak, M. (eds.) EGC 2005. LNCS, vol. 3470, pp. 111-121. Springer, Heidelberg (2005)

12. Bobroff, N., Fong, L., Kalayci, S., Martinez, J.C., Rodero, I., Sadjadi, S.M., Villegas, D.: Enabling Interoperability among Meta-Schedulers. In: 8th IEEE International Symposium on Cluster Computing and the Grid, Lyon, France (2008)

13. Parallel Workloads Archive, http://www.cs.huji.ac.il/labs/parallel/workload/

14. Grid Workloads Archive, http://gwa.ewi.tudelft.nl/

15. https://forge.gridforum.org/sf/projects/gsa-rg

16. http://latinamericangrid.org/

17. http://www.ogf .org/documents/GFD.56.pdf 